\title{
EMBALAGENS E RECOBRIMENTO EM LICHIAS (Litchi chinensis Sonn.) ARMAZENADAS SOB CONDIÇÕES NÃO CONTROLADAS
}

\author{
Packing and covering in lychee (Litchi chinensis Sonn.) stored under uncontrolled conditions
}

\author{
Rafaella Araújo Zambaldi Lima1, Celeste Maria Patto de Abreu², Simone Abreu Asmar², \\ Angelita Duarte Corrêa ${ }^{2}$, Custódio Donizete dos Santos ${ }^{2}$
}

\begin{abstract}
RESUMO
A lichia (Litchi chinensis Sonn.) é um fruto subtropical de alto potencial comercial devido ao seu sabor levemente acidificado, excelente aroma, alto valor nutritivo e atrativa cor vermelha da casca. Ainda no campo, a cor da casca altera-se facilmente, tornandose escurecida em resposta a estresses (alterações da umidade relativa e ataque de patógenos). Após colhida, a casca da lichia perde rapidamente sua cor vermelha. $\mathrm{O}$ escurecimento da casca tem sido atribuído a rápida degradação da antocianina, assim como: à rápida perda de umidade pelo fruto e à atividade de enzimas oxidativas como polifenoloxidases e peroxidases. Dada essa alta perecibilidade, o controle do escurecimento é fundamental para o aumento na vida útil pós-colheita, visando ao mercado interno e à exportação de frutas. Avaliou-se, neste trabalho, o comportamento pós-colheita de lichias da cv. Bengal quanto ao escurecimento da casca e às modificações relacionadas com a qualidade da polpa de lichias submetidas a diferentes embalagens: bandeja recoberta com filme de polietileno de baixa densidade com e sem perfurações e frutos recobertos com película de fécula de mandioca (3\%). Os frutos foram mantidos em temperatura ambiente $\left(25,1^{\circ} \mathrm{C} \pm 1,5^{\circ} \mathrm{C} ; 69,3 \% \pm 8,62 \% \mathrm{UR}\right)$ e armazenados por seis dias. Foram realizadas análises físicas, químicas e bioquímicas no dia 0 e a cada 2 dias, até osexto dia de armazenamento. A embalagem recoberta com filme de polietileno perfurado apresentou-se eficiente na redução da perda de massa, escurecimento da casca e na manutenção do teor de antocianina da casca de lichias durante seis dias de armazenamento, em temperatura ambiente.
\end{abstract}

Termos para indexação: Atmosfera modificada, antocianina, polifenoloxidase, peroxidase.

\section{ABSTRACT}

Lychee is a subtropical fruit of high commercial value, due to its slightly acid taste, excellent aroma, high nutritive value and attractive deep bright red color of its peel. Also in the field, skin color changes easily, becoming dark in response to stress (changes in relative humidity and the incidence of pathogens). Harvested lychee fruit rapidly loses its bright red skin colour. Peel browning of harvested lychee has largely been attributed to the rapid degradation of the anthocyanin pigments as well as the rapid loss of moisture from the fruit and the activity of oxidative enzymes such polyphenoloxidase and peroxidases. Due to it high perishability, browning control is a fundamental process to the increase of post-harvest useful life aiming the home market and fruit exportation. This study evaluated the postharvest behavior of Litchi cv. Bengal fruits regarding pericarp browning and changes on lychee fruits pulp quality when different packing is applied: plastic trays covered with parafilm or solely parafilm, and fruits covered with cassava starch film (3\%). Lychee fruits were stored for six days at room temperature $\left(25.1^{\circ} \mathrm{C} \pm 1.5 ; 69.3 \% \mathrm{UR}\right)$. Physical, chemical and biochemical analyses were performed on day 0 and on every two days till the end of the storage period. The package covered with perforated polyethylene film was efficient in reducing weight loss, skin browning and maintenance of the anthocyanin content of the bark of leeches for six days of storage at room temperature.

Index terms: Modified atmosphere, anthocyanin, polyphenol oxidase, peroxidase.

(Recebido em 18 de junho de 2009 e aprovado em 5 de março de 2010)

\section{INTRODUÇÃO}

A lichia (Litchi chinensis Sonn.), um fruto tropical originário da China, possui um pericarpo vermelho brilhante atrativo que envolve uma polpa translúcida de grande valor nutritivo. Além da importância nutritiva a lichia vem, nos últimos anos, adquirindo grande destaque comercial, sendo uma boa alternativa financeira para os problemas que ocorrem com as culturas tradicionais.

O grande atrativo desse fruto é a coloração vermelha da casca, um dos mais importantes aspectos de qualidade. Porém, a lichia apresenta um problema póscolheita: a casca torna-se escura de 2 a 3 dias após ter sido colhida, prejudicando sua comercialização. Mesmo causando pouca alteração na qualidade da polpa, o escurecimento prejudica a aparência do fruto, requisito fundamental de qualidade na aquisição de um produto pelo consumidor, além do mercado internacional como o europeu, ser exigente quanto às importações de lichia.

O pigmento responsável pela atrativa cor vermelha é a antocianina, que é normalmente instável, especialmente

\footnotetext{
'Universidade Federal de Lavras/UFLA - Departamento de Química/DQI - Cx. P. 3037 - 37200-000 - Lavras, MG - rafazambaldi@hotmail.com 2Universidade Federal de Lavras/UFLA - Departamento de Química /DQI - Lavras, MG
} 
quando a hidrólise da molécula libera a antocianidina livre, um processo que ocorre enzimaticamente em produtos vegetais após a colheita. As antocianidinas são prontamente oxidáveis por enzimas presentes no tecido vegetal, como as fenoloxidases e peroxidases, produzindo compostos marrons (Simpson et al., 1976).

O escurecimento enzimático em tecido vegetal é causado principalmente pela oxidação de fenóis endógenos e a subsequente polimerização não enzimática da $o$-quinona, formando pigmentos escuros denominados melaninas (Francis, 1989).

Com a perda de água dos frutos, durante o armazenamento, há uma descompartimentalização das células promovendo o contato das enzimas e seus substratos, causando reações indesejáveis, como o caso do escurecimento da casca da lichia. Portanto, procedimentos capazes de diminuir a perda de massa dos frutos e minimizar ou evitar o contato do fruto com o oxigênio são úteis na prevenção do escurecimento.

Desenvolveu-se, este trabalho, para avaliar o efeito de atmosferas modificadas no escurecimento de lichias armazenadas, por seis dias, em temperatura ambiente, pela realização de análises químicas e bioquímicas de compostos responsáveis pelo escurecimento da casca.

\section{MATERIAL E MÉTODOS}

\section{Material}

Lichias cv. Bengal, safra 2008, foram adquiridas de um pomar comercial da região de Lavras, MG.

Os frutos, após colhidos, foram levados para o Laboratório de Bioquímica do Departamento de Química da Universidade Federal de Lavras onde foi realizada a seleção, de acordo com a uniformidade da cor, tamanho e ausência de defeitos.

\section{Métodos}

Em todos os tratamentos, os frutos foram acondicionados em bandejas de tereftalato de polietileno (PET), em número de 15 por bandeja, e armazenados à temperatura ambiente $\left(25,1^{\circ} \mathrm{C} \pm 1,5^{\circ} \mathrm{C} ; 69,3 \% \pm 8,62 \% \mathrm{UR}\right)$ por seis dias.

- Frutos sem embalagem (C);

- Frutos recobertos com filme de polietileno de baixa densidade $\left(10 \mu \mathrm{m}, 60 \mathrm{TPO}_{2}, 280 \mathrm{TPCO}_{2} \mathrm{e}<0,01 \mathrm{TPH}_{2} 0\right)$, sem perfuração $(\mathrm{F})$;

- Frutos recobertos por filme de polietileno de baixa densidade com 15 perfurações distribuídas em três colunas e cinco linhas, com $2,9 \mathrm{~cm}$ de espaçamento entre colunas e $2,25 \mathrm{~cm}$ entre linhas. $\mathrm{O}$ diâmetro dos furos foi de $1,0 \mathrm{~mm}(\mathrm{FP})$;
- Frutos imersos por 1 minuto em solução de fécula de mandioca $(3 \%)$ e secas à temperatura ambiente (FM). A solução de fécula foi preparada aquecendo-se a mistura $1000 \mathrm{ml}$ de água e $30 \mathrm{~g}$ de fécula de mandioca comercial, até $70^{\circ} \mathrm{C}$ (Reis et al., 2006).

As análises foram realizadas em 4 tempos diferentes: dia da colheita, 2, 4 e 6 dias após a colheita.

$\mathrm{O}$ experimento foi conduzido em delineamento inteiramente casualizado, em esquema fatorial $(4 \times 4) \times 4$, com 4 tratamentos, 4 tempos de análise e 4 repetições. Os resultados foram submetidos à análise de variância e regressão.

\section{Perda de massa}

Os frutos, nos dia de análise, foram pesados em balança analítica, e os resultados expressos em porcentagem de perda de massa fresca.

\section{Escurecimento visual da casca}

Foi determinado, subjetivamente, pela adoção da seguinte escala (Zhang et al., 2004):

- Frutos com o pericarpo completamente vermelho valor um (1);

- Frutos com pericarpo $75 \%$ vermelho e $25 \%$ escuro valor dois (2);

- Frutos com pericarpo $50 \%$ vermelho e $50 \%$ escuro valor três (3), limite de comercialização;

- Frutos com pericarpo $25 \%$ vermelho e $75 \%$ escuro valor quatro (4), rejeitado comercialmente;

- Frutos com pericarpo totalmente escuro - valor cinco (5).

\section{Determinação do conteúdo de antocianina}

Utilizou-se o método de Fuleki \& Francis (1968). O conteúdo total de antocianina (mg. $100 \mathrm{~g}^{-1}$ de casca fresca) foi calculado em quantidades absolutas, com a utilização do coeficiente de extinção $(E=68,3)$ estabelecido para o pigmento predominante da lichia (cianidina 3-glucosídeo) em solvente alcoólico.

\section{Extração da peroxidase e polifenoloxidase}

Realizada com tampão fosfato 0,1 mol.L $\mathrm{L}^{-1}$ (pH 6,5), através de maceração realizada em banho de gelo, contendo $10 \%$ de polivinilpirrolidona (PVP) insolúvel. Em seguida, o homogenado foi filtrado e centrifugado a $10000 \mathrm{~g}$ durante 15 min, a $4{ }^{\circ} \mathrm{C}$ (Zauberman et al., 1991).

\section{Determinação da atividade da peroxidase e polifenoloxidase}

Realizada segundo Khan \& Robinson (1994). Utilizou-se como meio de reação: guaiacol $1 \%\left(\mathrm{v} \cdot \mathrm{v}^{-1}\right), \mathrm{H}_{2} \mathrm{O}_{2}$ 
$0,3 \%\left(\mathrm{v} \cdot \mathrm{v}^{-1}\right)$, extrato enzimático e tampão fosfato de sódio 0,05 mol. $\mathrm{L}^{-1} \mathrm{pH}$ 6,0. A reação foi acompanhada durante 2 minutos a $30{ }^{\circ} \mathrm{C}$ em espectrofotôme-tro. A atividade de peroxidase foi expressa em unidade (atividade capaz de alterar 0,001 de absorbância a $470 \mathrm{~nm}$ ) por minuto, por grama de matéria fresca (U.g $\left.{ }^{-1} \mathrm{MF}\right)$.

A atividade da polifenoloxidase foi realizada segundo Zauberman et al. (1991) utilizando-se catecol (0,1 mol.L-1 $)$ como substrato . A reação foi acompanhada durante 5 minutos, a $30{ }^{\circ} \mathrm{C}$ em espectrofotôme-tro. A atividade da polifenoloxidase foi expressa em unidade (atividade capaz de alterar 0,001 de absorbância a $395 \mathrm{~nm}$ ) por minuto, por grama de matéria fresca $\left(U \cdot g^{-1} \mathrm{MF}\right)$.

\section{RESULTADOS E DISCUSSÃO}

\section{Perda de massa}

Os tratamentos controle $(\mathrm{C})$ e fécula de mandioca $(\mathrm{FM})$ foram os que apresentaram maior perda de massa no final do período de armazenamento, porém o tratamento controle $(\mathrm{C})$ apresentou perda de massa mais rápida, alcançando 11,95\% no segundo dia após a colheita contra 4,14\% do tratamento FM (Figura 1). Esses mesmos foram diferentes dos frutos dos tratamentos F e FP que apresentaram as menores perdas de massa. Essa menor perda de massa, indica que as embalagens recobertas por filme de polietileno, perfurado ou não, evitaram uma maior perda de água.
A atmosfera modificada passiva contribuiu para reduzir o déficit de pressão de vapor d'água entre fatias de banana 'Maçã' e a atmosfera no interior da embalagem, minimizando a perda de massa (Melo et al., 2009).

Fontes et al. (1999) observaram que a perda de massa foi menor em lichias cv. Brewster, envoltas com filme de polietileno sem perfurações, atingindo $2 \%$ de perda, quando comparados aos frutos controle, até o completo escurecimento do pericarpo à temperatura ambiente.

Segundo Brown (1986), o escurecimento do pericarpo ocorre quando a perda de massa alcança 3-5\% do peso da fruta, enquanto Liang et al. (1998) concluiram que o mesmo ocorre com valores superiores à 7,6\%, da mesma forma, Wu et al. (1997) sugeriram que a perda de massa, para que o escurecimento ocorra, deve ser superior a $9 \%$ do peso da fruta. A grande variação nos valores sugeridos pode ocorrer devido a vários fatores, incluindo diferentes métodos de avaliação, condições de cultivo, cultivar, teor de água na colheita e tratamento antes da medição.

\section{Escurecimento visual}

A cor do pericarpo mudou gradativamente durante o armazenamento. Os frutos controle (C) perderam a cor vermelha rapidamente, atingindo a cor marrom, completamente, no sexto dia de armazenamento (Figura 2).



Figura 1 - Valores de perda de massa de lichias submetidas aos diferentes tratamentos C - controle, F - filme, FP - filme perfurado e FM - fécula de mandioca, durante seis dias de armazenamento à temperatura ambiente. DQI, UFLA, 2009. 
A maioria dos frutos do tratamento FM, no segundo dia de armazenamento, apresentou $50 \%$ da casca escura. Os frutos do tratamento $\mathrm{F}$ apresentaram-se, no segundo dia de armazenamento, sem nenhum escurecimento e, no quarto dia de armazenamento, poucos frutos continham manchas escuras na casca, assim como os frutos do tratamento FP.

Huang et al. (2005) verificaram que lichias da cv. Huaizhi tornaram-se marrons em apenas dois dias, quando mantidas à temperatura ambiente. Zhang et al. (2004), verificaram que o índice de escurecimento aumentou durante o armazenamento, ao mesmo tempo em que a concentração total de antocianina diminuiu marcadamente.

O escurecimento do pericarpo da lichia durante o armazenamento está associado, entre outros fatores, com a perda de água que aumenta com o tempo. Esse fato faz com que o pericarpo desidrate e escureça rapidamente. $\mathrm{O}$ fruto escuro é resultado da perda de integridade da membrana celular, um evento crucial na senescência do fruto.

As análises de escurecimento visual do pericarpo demonstraram que os frutos que apresentaram a maior perda de massa (C e FP) são também os que obtiveram maior escurecimento durante o armazenamento, confirmando a relação direta entre a desidratação e o escurecimento do pericarpo.

\section{Antocianina}

$\mathrm{O}$ aumento na perda de massa dos frutos coincide com a queda na concentração de antocianina que foi mais acentuada para os frutos componentes dos tratamentos C e FM (Figura 3). O conteúdo médio de antocianina, no dia da colheita, variou de $86,7 \mathrm{mg} 100 \mathrm{~g}^{-1}$ a $94,1 \mathrm{mg} .100 \mathrm{~g}^{-1}$ na casca fresca, valores semelhantes aos encontrados na literatura. No sexto dia, esse valor caiu em 50,1\% para os frutos do tratamento C, 54,4\% para os frutos do tratamento FM, 22,3\% para o tratamento FP e $20,2 \%$ para o tratamento F. O fato dos frutos desses dois tratamentos terem apresentado menor queda no conteúdo de antocianina pode estar relacionado com a menor disponibilidade de $\mathrm{O}_{2}$ no interior da embalagem, menor perda de água, menor descompartimentalização das células e, consequentemente, menor atividade enzimática.

O índice de escurecimento da lichia aumenta durante o armazenamento à temperatura ambiente, assim como a concentração de antocianina diminui marcadamente (Zhang et al., 2004).

\section{Polifenoloxidase (PPO)}

Os frutos do tratamento $\mathrm{C}$ apresentaram maior atividade enzimática com relação aos demais tratamentos (Tabela 1).

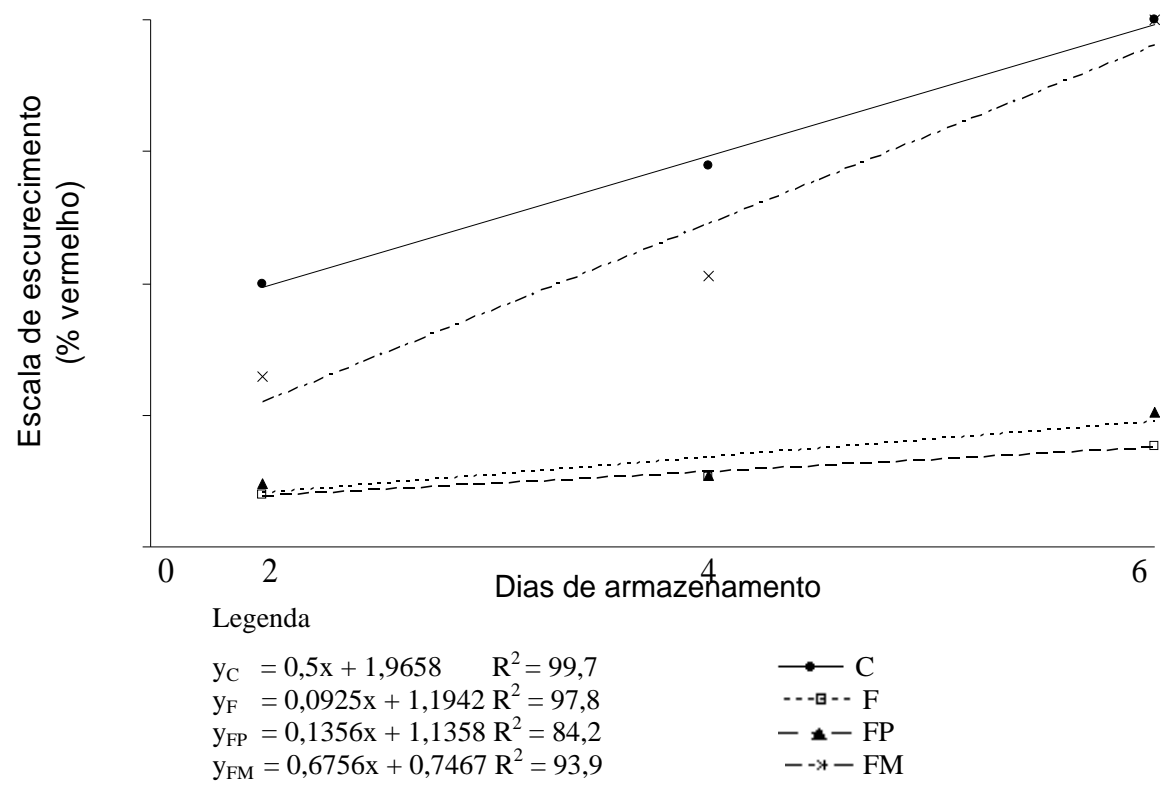

Figura 2 - Valores do escurecimento visual da casca de lichias submetidas aos diferentes tratamentos $\mathrm{C}$ - controle, $\mathrm{F}$ - filme, FP - filme perfurado e FM - fécula de mandioca, durante seis dias de armazenamento, à temperatura ambiente. DQI, UFLA, 2009. 
O tratamento FM, quando comparado aos tratamentos F e FP, foi menos eficiente na manutenção do peso do fruto. A maior perda de massa dos frutos desse tratamento pode ter ocasionado maior descompartimentação celular com consequente aumento na atividade enzimática durante o armazenamento dos frutos deste tratamento.

Os tratamentos F e FP apresentaram valores de atividade enzimática semelhantes, a atividade aumentou linearmente até o final do período de armazenamento (Figura 4).

Lin et al. (1988) demonstraram um rápido aumento na atividade da PPO durante as primeiras 48 horas de armazenamento e Huang et al. (1990), observaram que durante o armazenamento, a concentração de antocianina diminuiu, enquanto a atividade da polifenoloxidase aumentou.

\section{Peroxidase (POD)}

A atividade da POD aumentou para todos os tratamentos, durante todo o período de armazenamento (Figura 5). Os frutos controle foram os que apresentaram maior atividade, seguido pelos frutos do tratamento FM (Tabela 2). Esse fato pode ser explicado pelo maior dessecamento do pericarpo ocorrido nos frutos desses tratamentos. Os frutos que pertenciam aos tratamentos $F$ e FP apresentaram as mais baixas atividades enzimáticas

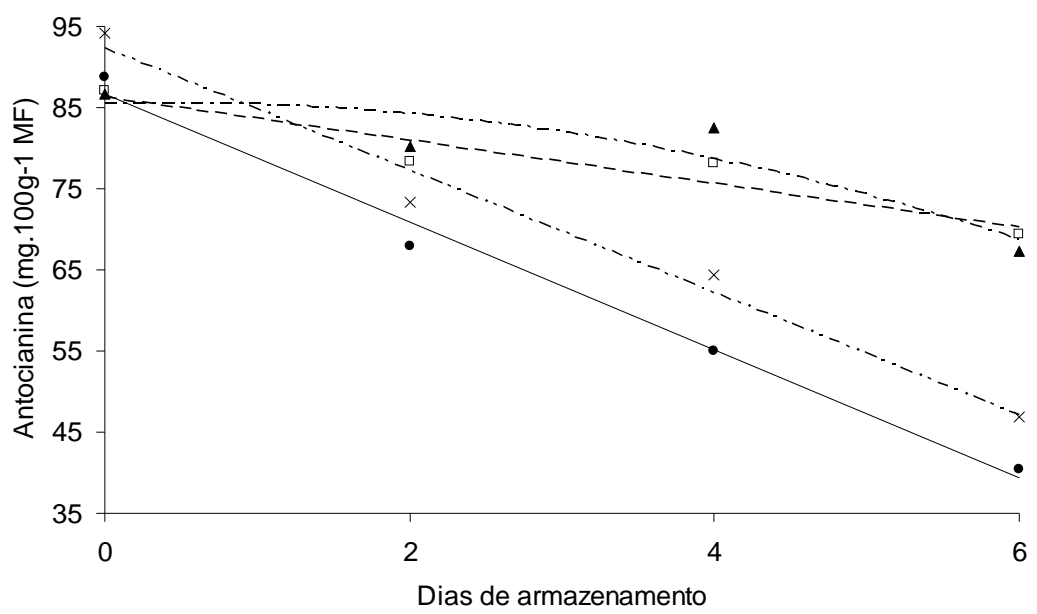

Legenda

$$
\begin{aligned}
& \mathrm{y}_{\mathrm{C}}=-7,907 \mathrm{x}+86,731 \quad \mathrm{R}^{2}=98,9 \quad \longrightarrow \mathrm{C} \\
& \begin{array}{lll}
y_{F}=-2,6605 x+86,184 R^{2}=91,0 & & --\mathrm{E} \\
y_{F P}=-0,5513 x^{2}+0,5275 x+85,375 & R^{2}=83,6 & -\star-F P
\end{array} \\
& \mathrm{y}_{\mathrm{FM}}=-7,536 \mathrm{x}+92,318 \quad \mathrm{R}^{2}=98,0 \quad--*-\mathrm{FM}
\end{aligned}
$$

Figura 3 - Valores de antocianina da casca de lichias submetidas aos diferentes tratamentos C - Controle, F - filme, FP - filme perfurado e FM - fécula de mandioca, durante seis dias de armazenamento em temperatura ambiente. DQI, UFLA, 2009.

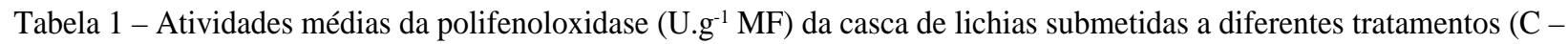
Controle, F - filme, FP - filme perfurado e FM - fécula de mandioca), durante seis dias de armazenamento à temperatura ambiente. DQI, UFLA, 2009.

\begin{tabular}{ccccc}
\hline \multirow{2}{*}{ Tratamentos } & \multicolumn{4}{c}{ Dias de armazenamento } \\
\cline { 2 - 5 } & 0 & 2 & 4 & 6 \\
\hline C & $105,43 \mathrm{a}$ & $206,83 \mathrm{a}$ & $308,23 \mathrm{a}$ & $409,63 \mathrm{a}$ \\
F & $90,50 \mathrm{a}$ & $168,50 \mathrm{~d}$ & $246,50 \mathrm{~d}$ & $324,50 \mathrm{~d}$ \\
FP & $81,98 \mathrm{a}$ & $178,38 \mathrm{c}$ & $274,78 \mathrm{c}$ & $371,18 \mathrm{c}$ \\
FM & $93,00 \mathrm{a}$ & $190,20 \mathrm{~b}$ & $287,40 \mathrm{~b}$ & $384,60 \mathrm{~b}$ \\
\hline
\end{tabular}

As médias seguidas da mesma letra, na coluna, não diferem entre si, à 5\% de probabilidade, pelo teste de Tukey. 
da POD.. Segundo Pinheiro et al. (2009), a atividade da peroxidase aumentou com o decorrer do tempo de armazenamento de abacates minimamente processados.
Zhang et al. (2004) observaram alta atividade da peroxidase $\left(8,5 \times 10^{3} \mathrm{U} \cdot \mathrm{min}^{-1} \cdot \mathrm{g}^{-1} \mathrm{MF}\right)$ para lichias $\mathrm{cv}$. Huaizhi, no quarto dia de armazenamento, à temperatura de $28^{\circ} \mathrm{C}$.

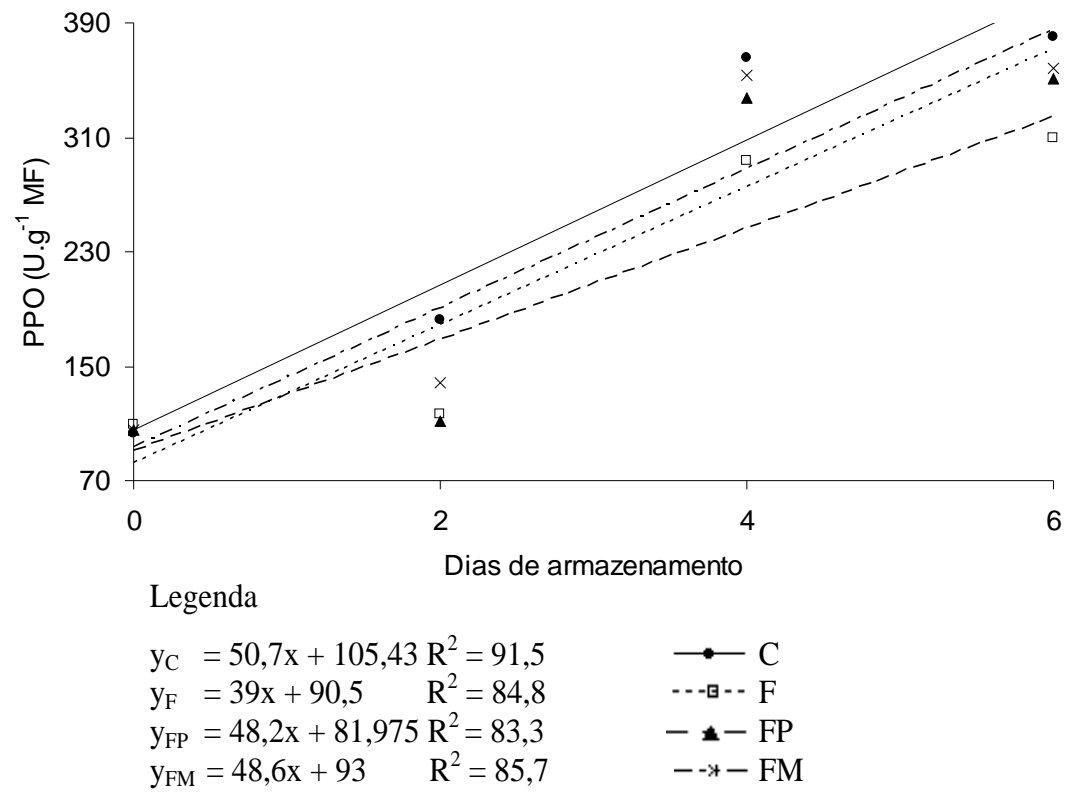

Figura 4 - Valores de atividade da polifenoloxidase da casca de lichias submetidas aos diferentes tratamentos C Controle, F - filme, FP - filme perfurado e FM - fécula de mandioca, durante seis dias de armazenamento à temperatura ambiente. DQI, UFLA, 2009.

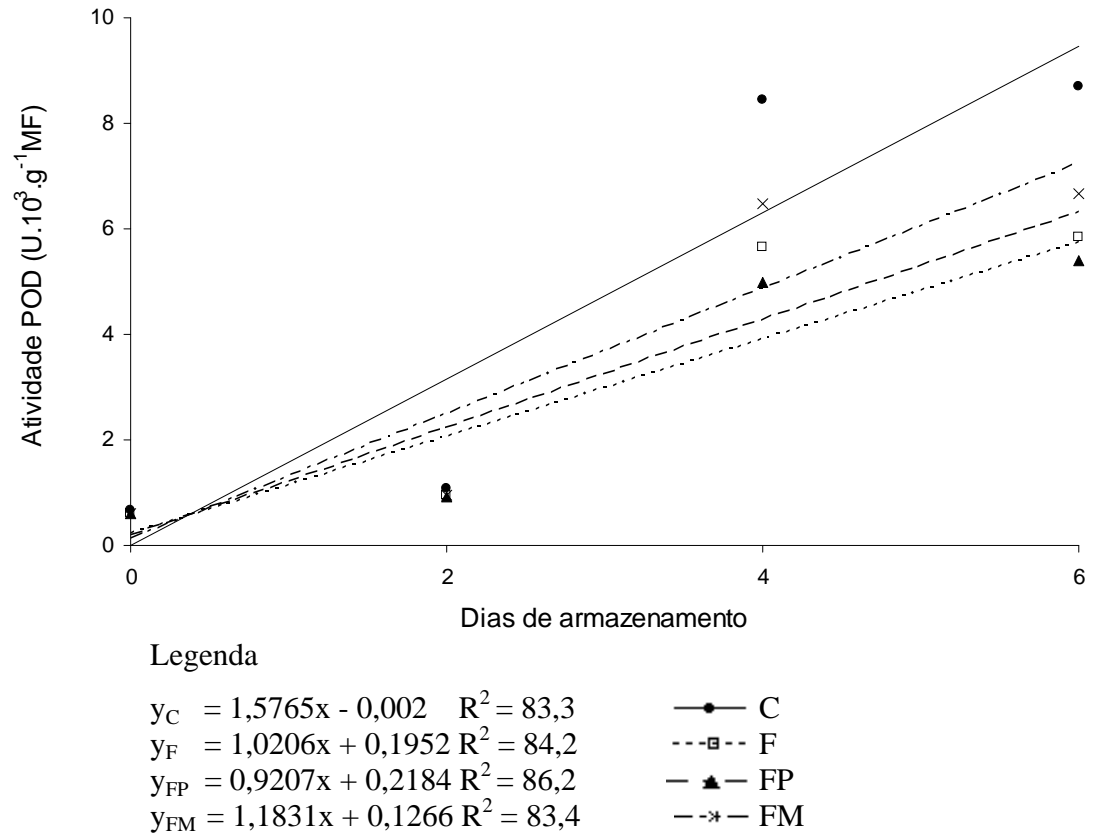

Figura 5 - Valores de atividade da peroxidase da casca de lichias submetidas aos diferentes tratamentos $\mathrm{C}$ - Controle, $\mathrm{F}$ - filme, FP - filme perfurado e FM - fécula de mandioca, durante seis dias de armazenamento à temperatura ambiente. DQI, UFLA, 2009. 
Tabela 2 - Atividades médias da peroxidase $\left(\mathrm{U} \cdot \mathrm{g}^{-1} \mathrm{MF}\right) \mathrm{da}$ casca de lichias submetidas a diferentes tratamentos $(\mathrm{C}-$ Controle, F - filme, FP - filme perfurado e FM - fécula de mandioca), durante seis dias de armazenamento à temperatura ambiente. DQI, UFLA, 2009.

\begin{tabular}{ccccc}
\hline \multirow{2}{*}{ Tratamentos } & \multicolumn{4}{c}{ Dias de armazenamento } \\
\cline { 2 - 5 } & 0 & 2 & 4 & 6 \\
\hline C & $0,00 \mathrm{a}$ & $3,15 \mathrm{a}$ & $6,30 \mathrm{a}$ & $9,48 \mathrm{a}$ \\
F & $0,20 \mathrm{a}$ & $2,24 \mathrm{c}$ & $4,28 \mathrm{c}$ & $6,56 \mathrm{c}$ \\
FP & $0,22 \mathrm{a}$ & $2,06 \mathrm{c}$ & $3,90 \mathrm{c}$ & $5,74 \mathrm{~d}$ \\
FM & $0,13 \mathrm{a}$ & $2,50 \mathrm{~b}$ & $4,86 \mathrm{~b}$ & $7,23 \mathrm{~b}$ \\
\hline
\end{tabular}

As médias seguidas da mesma letra, na coluna, não diferem entre si, à $5 \%$ de probabilidade, pelo teste de Tukey.

\section{CONCLUSÕES}

A embalagem recoberta com filme de polietileno perfurado apresentou-se eficiente na redução da perda de massa, escurecimento da casca e na manutenção do teor de antocianina da casca de lichias durante seis dias de armazenamento, à temperatura ambiente.

A utilização de solução de fécula de mandioca para a formação de película protetora, não foi eficiente para prevenir ou retardar o escurecimento da casca da lichia.

\section{AGRADECIMENTOS}

Ao CNPq e à CAPES pelo apoio financeiro.

\section{REFERÊNCIAS BIBLIOGRÁFICAS}

BROWN, B.I. Postharvest handling and storage of lychees. In: NATIONAL LYCHEE SEMINAR, 1., 1986, Nambour. Proceedings... Nambour: Sunshine Coast Tropical Fruits Association, 1986. p.77-78.

FONTES, V.L.; MOURA, M.A.; VIEIRA, G.; FINGER, F.L. Efeito de filmes plásticos e temperatura de armazenamento na manutenção da cor do pericarpo de lichia. Revista Brasileira de Armazenamento, Viçosa, v.24, n.1, p.56-59, 1999.

FRANCIS, F.J. Food colorants: anthocyanins. Critical Review of Food Science and Nutrition, Boca Raton, v.28, n.4, p.273-314, 1989.

FULEKI, T.; FRANCIS, F.J. Quantitative methods for anthocyanins: extraction and determination of total anthocyanin in cranberries. Journal of Food Science, Chicago, v.33, n.1, p.72-83, Jan./Feb. 1968.
HUANG, S.; HART, H.; LEE, H.; WICKER, L. Enzimatic and colour changes during post-harvest storage of lychee fruit. Journal of Food Science, Chicago, v.55, n.6, p.1762-1763, Nov./Dec. 1990.

HUANG, X.M.; WANG, H.C.; YUAN, W.G.; LU, J.M.; YIN, J.H.; LUO, S.; HUANG, H.B. A study of rapid senescence of detached litchi: roles of water loss and calcium. Postharvest Biology and Technology, Amsterdam, v.36, n.2, p.177-189, May 2005.

KHAN, A.A.; ROBINSON, D.S. Hydrogen donor specificity of mango isoperoxidases. Food Chemistry, Oxford, v.49, n.4, p.407-410, Dec. 1994.

LIANG, H.H.; JI, Z.L.; HUANG, X.Y. Study on the techniques of package and storage for litchi fruit stored in the room temperature. Journal of Fruit Science, v.15, n.2, p.158-163, 1998.

LIN, Z.F.; LI, S.S.; ZHANG, D.L.; LIN, G.Z.; LI, Y.B.; LIU, S.X.; CHEN, M.D. The changes of pigments, phenolics contents and activities of polyphenol oxidase and phenylalanine ammonia-lyase in pericarp of postharvest litchi fruit. Acta Botanica Sinica, Beijing, v.30, p.40-45, 1988.

MELO, A. A. M.; VILAS BOAS, E. V. de B.; JUSTO, C. F. Uso de aditivos químicos para a conservação póscolheita de banana 'Maçã' minimamente processada. Ciência e Agrotecnologia, Lavras, v.33, n.1, p.228-223, Jan./Fev., 2009.

PINHEIRO, A. C. M.; VILAS BOAS, E. V. de B.; SILVA, L. C. e; ALVES, A. de P.; LA SELVA, M.; CHITARRA, A. B. Quality of fresh-cut avocado (Persea americana Mill.) stored under different temperatures. Ciência e Agrotecnologia, Lavras, v.33, n.4, p.1095-1102, Jul./Ago., 2009.

REIS, K.C.; ELIAS, H.H.S.; LIMA, L.C.O.; SILVA, J.D.; PEREIRA, J. Pepino japonês (Cucumis sativus L.) submetido ao tratamento com fécula de mandioca. Ciência e Agrotecnologia, Lavras, v.30, n.3, p.487-493, 2006.

SIMPSON, K.L.; LEE, T.C.; RODRIGUE, J.H.; CHICHESTER, C.O. Metabolism in senenscent and stored tissue. In: GOODWIN, T.W. (Ed.). Chemistry and biochemistry of plant pigment. 2.ed. New York: Academic, 1976. v.1, p.128-155. 
WU, Z.X.; SU, M.X.; CHEN, W.X. Research advance on mechanism of litchi browning. In: CHINA AGRICULTURAL PRODUCTS STORING AND PROCESSING TECHNICAL ANNALS, 1997, Beijing. Proceedings... Beijing: China Agricultural University, 1997. p.294-302.

ZAUBERMAN, G.; RONEN, R.; AKERMAN, M.; WEKSLER, A.; ROT, I.; FUCHS, Y. Postharvest retention of the red colour of litchi fruit pericarp. Scientia

Horticulturae, Amsterdam, v.46, n.1/2, p.89-97, June 1991.

ZHANG, Z.Q.; PANG, X.Q.; YANG, C.; JI, Z.L.; JIANG, Y.M. Purification and structural analysis of anthocyanins from litchi pericarp. Food Chemistry, Oxford, v.84, n.4, p.601-604, Mar 2004. 